\title{
Inhibition of phosphodiesterase 4 modulates cytokine induction from toll like receptor activated, but not rhinovirus infected, primary human airway smooth muscle
}

David Van Ly ${ }^{1,2^{*}}$, Monique De Pedro ${ }^{1,2}$, Peter James ${ }^{1,2}$, Lucy Morgan ${ }^{3}$, Judith L Black ${ }^{1,2}$, Janette K Burgess ${ }^{1,2}$ and Brian GG Oliver ${ }^{1,2}$

\begin{abstract}
Background: Virus-induced exacerbations of Chronic Obstructive Pulmonary Disease (COPD) are a significant health burden and occur even in those receiving the best current therapies. Rhinovirus (RV) infections are responsible for half of all COPD exacerbations. The mechanism by which exacerbations occur remains undefined, however it is likely to be due to virus-induced inflammation. Given that phophodiesterase $4\left(\mathrm{PDE}_{4}\right)$ inhibitors have an anti-inflammatory effect in patients with COPD they present a potential therapy prior to, and during, these exacerbations.

Methods: In the present study we investigated whether the $\mathrm{PDE}_{4}$ inhibitor piclamilast $\left(10^{-6} \mathrm{M}\right)$ could alter RV or viral mimetic $(5 \mu \mathrm{g} / \mathrm{mL}$ of imiquimod or poly $\mathrm{l}: \mathrm{C})$ induced inflammation and RV replication in primary human airway smooth muscle cells (ASMC) and bronchial epithelial cells (HBEC). The mediators IL-6, IL-8, prostaglandin $E_{2}$ and CAMP production were assayed by ELISA and RV replication was assayed by viral titration.

Results: We found that in ASMCs the TLR3 agonist poly I:C induced IL-8 release was reduced while induced IL-6 release by the TLR7/8 agonist imiquimod was further increased by the presence of piclamilast. However, in RV infected ASMCs, virus replication and induced mediator release were unaltered by piclamilast, as was also found in HBECs. The novel findings of this study reveal that although PDE inhibitors may not influence RV-induced cytokine production in ASMCs and replication in either ASMCs or HBECs, they have the capacity to be anti-inflammatory during TLR activation by modulating the induction of these chemotactic cytokines.
\end{abstract}

Conclusion: By extrapolating our in vitro findings to exacerbations of COPD in vivo this suggests that $\mathrm{PDE}_{4}$ inhibitors may have beneficial anti-inflammatory properties when patients are infected with bacteria or viruses other than RV.

Keywords: Piclamilast, Rhinovirus, Poly I: C, Formoterol, Imiquimod, Phosphodiesterase 4, COPD, Airway smooth muscle

\section{Background}

Chronic Obstructive Pulmonary Disease (COPD) is characterised by irreversible airflow obstruction, inflammation and a progressive decline in lung function [1] which is increasing in prevalence and predicted to be the third leading cause of death worldwide by 2030. Exacerbations of COPD are the major cause of morbidity

\footnotetext{
* Correspondence: dvan7422@gmail.com

'Woolcock Institute of Medical Research, Sydney, Australia

${ }^{2}$ Respiratory Research Group, Discipline of Pharmacology, The University of Sydney, Sydney, New South Wales, Australia

Full list of author information is available at the end of the article
}

and mortality and are associated with accelerated decline in lung function and progression of the disease [2]. Approximately $50 \%$ of all COPD exacerbations are due to rhinovirus (RV) infections and, therefore, are a major problem for people with COPD $[2,3]$.

The mechanism by which RV causes exacerbations is not fully understood, but is thought to be related to RVinduced activation of the innate immune system and subsequent inflammation [4]. The innate immune system detects pathogens through the expression of pattern recognition receptors (PRR), of which toll-like receptors (TLR) are perhaps the best characterized [5]. Human

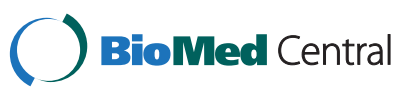


bronchial epithelial cells (HBEC), fibroblasts and airway smooth muscle cells (ASMC) express TLRs and are involved in the innate immune response to viral infections in the lungs [6]. During viral infections these cells can detect RV as well as other viruses through the presence of double stranded RNA (dsRNA) via TLR3, Retinoic Acid-Inducible Gene 1 (RIG-I) and Melanoma Differentiation-Associated protein 5 (MDA-5); and single stranded RNA (ssRNA) via TLR7/8 $[7,8]$.

In the healthy airways, HBECs form the barrier between the external environment and the underlying fibroblasts and ASMCs and represent the initial point for RV infection [9]. However in COPD the bronchial epithelial layer is often compromised allowing submucosal infection to occur. Of these airway cells, ASMCs directly control bronchomotor tone and therefore make them primary drug targets for the relief of an exacerbation by $\beta_{2}$ adrenoreceptor $\left(\beta_{2}-\mathrm{AR}\right)$ agonists. Research has shown that RV has the ability to infect ASMCs $[10,11]$, and ASMCs can also contribute to the local immune environment through the production of a wide variety of immunomodulatory factors such as interleukin (IL) -6 and -8 and regulating airway contraction $[10,12,13]$. Therefore ASMCs are undoubtedly an important contributor and direct modulator during respiratory exacerbations and investigations into these cells may offer novel therapies for exacerbations of COPD.

cAMP is a secondary signalling messenger that is able to alter the transcription of genes through the binding and activation of cAMP response element (CRE) binding protein (CREB) to CRE sequences present in the promoter region of genes [14]. IL-6 and cyclo-oxygenase (COX)-2 promoter regions both contain CRE sequences and cAMP mobilizers such as $\beta_{2}$-AR agonists that induce cAMP could potentially affect these mediators [15-17]. Using IL-6 promoter constructs we have previously shown RV-induced inflammation is mediated in part via cAMP signalling [10] and others have shown that RV infection increases the activity of adenyly cyclase (the enzyme which produces cAMP) therefore sensitizing the cAMP pathway [18]. Furthermore, a synergistic increase in RV-induced IL-6 occurs in the presence of cAMP mobilizing agents such as $\beta_{2}$-AR agonists [19]. Not all genes containing a CRE are pro-inflammatory (e.g. IL-10) and not all RV-induced mediators are the result of cAMP signalling. For example IL-8, which is also induced by RV in vitro in ASMCs, has been shown to be poorly induced in response to cAMP, suggesting its transcription may not be directly regulated by the CREB protein $[20,21]$.

Phosphodiesterases (PDEs) regulate cAMP signalling by hydrolysis of cAMP and we have previously shown cAMP is exquisitely regulated by $\mathrm{PDE}_{4}$ in airway cells [22]. Inhibitors of $\mathrm{PDE}_{4}$ have now been developed and the $\mathrm{PDE}_{4}$ inhibitor roflumilast has been approved as anti-inflammatory therapy for the treatment of COPD. Roflumilast reduces sputum neutrophil (35\%) and eosinophil (50\%) numbers [23] and decreases the number of COPD exacerbations [8]. A similar analogue, cilomilast, has also been shown to decrease basal levels of the immunomodulatory cytokine IL-8 in HBECs obtained from patients with bronchiolitis obliterans syndrome suggesting their anti-inflammatory effects in inflammatory diseases maybe by regulating immunomodulatory cytokines via cAMP pathways directly or indirectly [24].

The main anti-inflammatory therapy used in the treatment of COPD is corticosteroids which are often used in combination with $\beta_{2}$-AR agonists. Recently the efficacy of this therapy was evaluated in a large randomised control trial involving 6112 COPD patients. It was found that the use of long-acting $\beta_{2}$-AR agonists and inhaled corticosteroids in combination resulted in significantly fewer exacerbations and improved health status and lung function, as compared with patients given a placebo [25]. However, corticosteroids were associated with an increased incidence of pneumonia and subsequent death, highlighting the need for better anti-inflammatory medications for the treatment of COPD.

Piclamilast is a $\mathrm{PDE}_{4}$ inhibitor with common structural and in vitro pharmacological characteristics to roflumilast [26]. We hypothesized that $\mathrm{PDE}_{4}$ inhibition would modulate virus-induced mediator release since many responses of ASMCs to virus infection are regulated by cAMP, and as a result decrease viral replication. The aim of this study was to investigate whether piclamilast could modulate the production of virus-induced mediators (both immunomodulatory cytokines such as IL-6 and -8 and anti-viral cytokines such as interferons (IFNs)) and virus replication in ASMCs, an important cell of the airways. Results of the study will deduce whether $\mathrm{PDE}_{4}$ inhibition may have potential suitability in the treatment of virus-induced exacerbations of COPD.

\section{Methods}

\section{Cell culture}

Primary HBECs and ASMCs were isolated from macroscopically healthy bronchial tissue obtained from patients (see Table 1 for demographics) undergoing resections or transplantations as previously described [11]. Ethical approval for all experiments involving the use of human lung tissue was provided by The University of Sydney Human Ethics Committee and the Sydney South West Area Health Service, and written informed consent was obtained. ASMCs were cultured in Dulbecco's Modified Eagle's Medium (DMEM) (Sigma-Aldrich, Castle Hill, Australia) supplemented with $10 \%(\mathrm{v} / \mathrm{v})$ foetal bovine serum (FBS) (10\% FBS/DMEM), 20U/mL penicillin, 
Table 1 Demographic data of study patients

\begin{tabular}{|c|c|c|c|c|c|}
\hline Patient & Cell & Sex & Age & Disease & Sample \\
\hline 1 & ASMC & Female & 27 & Healthy volunteer & Bronchoscopy \\
\hline 2 & ASMC & Female & 21 & Healthy volunteer & Bronchoscopy \\
\hline 3 & ASMC & Male & 56 & Metastatic Melanoma & Resection \\
\hline 4 & ASMC & Female & 29 & $\begin{array}{l}\text { Pulmonary } \\
\text { Hypertension }\end{array}$ & Transplant \\
\hline 5 & ASMC & Female & 43 & Emphysema & Transplant \\
\hline 6 & ASMC & Female & 56 & Emphysema & Transplant \\
\hline 7 & ASMC & Female & 53 & Pulmonary Fibrosis & Transplant \\
\hline 8 & ASMC & Male & 66 & NSCCA & Resection \\
\hline 9 & ASMC & Male & 57 & Pulmonary Fibrosis & Transplant \\
\hline 10 & ASMC & Female & 59 & Adeno Ca & Resection \\
\hline 11 & ASMC & Male & 75 & NSCCA & Resection \\
\hline 12 & ASMC & Female & 41 & $\mathrm{Ca}$ & Resection \\
\hline 13 & ASMC & Female & 62 & Squamous Cell Ca & Resection \\
\hline 14 & ASMC & Male & 67 & NSCCA & Resection \\
\hline 15 & ASMC & Female & 15 & $\begin{array}{l}\text { Pulmonary } \\
\text { Hypertension }\end{array}$ & Transplant \\
\hline 16 & ASMC & Male & 58 & Emphysema & Transplant \\
\hline 17 & ASMC & Female & 68 & NSCCA & Resection \\
\hline 18 & ASMC & Male & 61 & NSCCA & Resection \\
\hline 19 & ASMC & Male & 55 & Emphysema & Transplant \\
\hline 20 & ASMC & Male & 54 & NSCCA & Resection \\
\hline 21 & ASMC & Male & 67 & NSCCA & Resection \\
\hline 22 & ASMC & Male & 63 & NSCCA & Resection \\
\hline 23 & ASMC & Female & 68 & Adeno Ca & Resection \\
\hline 24 & ASMC & Female & 60 & Emphysema & Transplant \\
\hline 25 & ASMC & Female & 62 & Emphysema & Transplant \\
\hline 26 & ASMC & Female & 73 & NSCCA & Resection \\
\hline 27 & ASMC & Female & 57 & Emphysema & Transplant \\
\hline 28 & ASMC & Female & 50 & Pulmonary Fibrosis & Transplant \\
\hline 29 & ASMC & Male & 67 & Emphysema & Transplant \\
\hline 30 & ASMC & Female & 78 & $\mathrm{Ca}$ & Resection \\
\hline 31 & ASMC & Female & 51 & Emphysema & Transplant \\
\hline 32 & ASMC & Female & 59 & Emphysema & Transplant \\
\hline 33 & HBEC & Female & 69 & Adeno Ca & Resection \\
\hline 34 & HBEC & Female & 45 & Adeno Ca & Resection \\
\hline 35 & HBEC & Male & 75 & NSCCA & Resection \\
\hline 36 & HBEC & Female & 58 & Emphysema & Transplant \\
\hline
\end{tabular}

Key: ASMC airway smooth muscle cells, HBEC human bronchial epithelial cells, NSCCA non small cell carcinoma, Ca cancer.

$20 \mathrm{~g} / \mathrm{mL}$ streptomycin and $2.5 \mathrm{~g} / \mathrm{mL}$ amphotericin $\mathrm{B}$ (Invitrogen, Mount Waverley, Australia) in $75 \mathrm{~cm}^{2}$ flasks. HBECs were cultured in selective bronchial epithelial growth medium (BEGM) (Clonetics, San Diego, California, USA) in $75 \mathrm{~cm}^{2}$ flasks. Cells were grown at $37^{\circ} \mathrm{C}$ in $5 \% \mathrm{CO}_{2}$ until confluent at which point they were passaged further. ASMCs were identified by morphology and staining for $\alpha$ smooth muscle actin [27] and used for experimentation between passages 4 and 8 . HBECs were used between passages 2-4. HeLa cells were maintained in 2\% FBS and grown in 10\% FBS supplemented in minimum essential medium (MEM), 0.025 M HEPES, $0.0375 \%(\mathrm{w} / \mathrm{v})$ sodium bicarbonate and $1 \%$ GlutaMAX ${ }^{\mathrm{st}}$ in $175 \mathrm{~cm}^{2}$ flasks (all from invitrogen). All cells used in this study were tested and found to be free of mycoplasma contamination.

\section{RV propagation}

Major group human RV serotype-16 was propagated in Ohio HeLa cells and purified using a 100,000 kDa molecular weight cut off (MWCO) filter as previously described [28]. RV concentrations were measured by using a tissue culture infectivity titration assay. Briefly, RV levels were determined by serially titrating log diluted concentrations of the cell free supernatant in quadruplicates on Ohio HeLa cells. Ohio HeLa cells were seeded at a concentration of $2 \times 10^{4}$ cells $/ \mathrm{mL}$ in 96 well plates $(150 \mu \mathrm{L} /$ well $)$ and then $50 \mu \mathrm{L}$ of supernatants with RV or control medium were added to the wells. The plates were rocked at $100 \mathrm{rpm}$ for 15 minutes at room temperature before being cultured for 5 days at $37^{\circ} \mathrm{C}$ at $5 \% \mathrm{CO}_{2}$. After 5 days of culture, the cytopathic effect was assessed and the tissue culture infectious dose $50\left(\mathrm{TCID}_{50}\right)$ of $\mathrm{RV}$ was quantified using the Spearman-Karber formulation.

\section{Drugs}

Formoterol, isoprenaline, 3-isobutyl-1-methylxanthine (IBMX), polyinosinic:polycytidylic acid (poly I:C) and imiquimod were all obtained from Sigma-Aldrich and piclamilast was obtained from Altana Pharma $\mathrm{GmbH}$ (Konstanz, Germany). Drugs were dissolved in dimethyl sulfoxide (DMSO) (Sigma-Aldrich) before dilution in treatment medium and vehicle controls contained the corresponding concentration of DMSO used in each experiment. Poly I:C and imiquimod were dissolved in phosphate buffer solution (PBS) (Invitrogen). The concentrations of the drugs used in this study were selected based upon previous published results [29] and/or maximal concentrations from dose response investigations.

\section{cAMP assay}

To assess cAMP concentration, ASMCs were stimulated for 5 or 30 minutes in the presence of the phosphodiesterase inhibitor IBMX $\left(10^{-5} \mathrm{M}\right)$ in hank's balanced salt solution (HBSS) (Invitrogen). In some experiments isoprenaline $\left(10^{-7} \mathrm{M}\right)$ was used as a positive control. The cells were then lysed in a solution of $\mathrm{H}_{2} \mathrm{O}$ with $0.03 \%$ (v/v) Tween-20 and 5 mM HEPES buffer by vigorous pipetting. The amount of cAMP in the lysate samples was quantified using an Alphascreen cAMP Assay Kit 
(Perkin Elmer, Massachusetts, USA) according to the manufacturer's instructions and read using an EnVision Multilabel Plate Reader.

\section{Drug treatment and infection of primary human ASMCs with RV-16 or drugs}

ASMCs or HBECs were seeded at $3.2 \times 10^{4}$ cells $/ \mathrm{mL}$ into 6 well plates in 10\% FBS/DMEM and grown for 3 days. For single drug treatments, the medium was replaced with $0.1 \%$ FBS/antibiotics/DMEM or BEGM and or drug/vehicle. Alternatively, for secondary drug treatments, piclamilast was added to the medium after 1 hour of incubation with the initial drug/vehicle and incubated for 24 hours at $37^{\circ} \mathrm{C}$ at $5 \% \mathrm{CO}_{2}$ prior to collection. For $\mathrm{RV}$ infection a cell count was carried out to determine the amount of RV needed to infect at a multiplicity of infection (MOI) of 1 . Some wells were then pretreated with drug/vehicle initially for 1 hour, then infected at an MOI of 1 with live RV in that medium and left for 1 hour at $37^{\circ} \mathrm{C}$ and $5 \% \mathrm{CO}_{2}$. Plates were rocked every 15 minutes to allow the virus to adsorb. The medium was removed, the cells washed with Hanks solution and $3 \mathrm{~mL} /$ well of 0.1\% FBS/antibiotics/DMEM or BEGM supplemented with the previous drug/vehicle was added. The plates were then incubated at $37^{\circ} \mathrm{C}$ and $5 \% \mathrm{CO}_{2}$ for 24 hours and supernatants were collected and stored at $-80^{\circ} \mathrm{C}$ prior to analysis using ELISA and RV titration assays.

\section{ELISA}

ELISA kits for the type III IFNs: IL-28B $\left(\lambda_{3}\right)$, IL-29 $\left(\lambda_{1}\right)$ and the cytokine IL- 8 were purchased from R\&D Systems (Minneapolis, USA); IL-6 was purchased from BD Biosciences (North Ryde, Australia); and prostaglandin $\mathrm{E}_{2} \quad\left(\mathrm{PGE}_{2}\right)$ was purchased from Cayman Chemicals (Michigan, USA). ELISAs were carried out according to the manufacturer's instructions. The detection limits of these assays were: $7.8125 \mathrm{pg} / \mathrm{mL}$ (IL-6), $15.625 \mathrm{pg} / \mathrm{mL}$ (IL-8) and $31.25 \mathrm{pg} / \mathrm{mL}$ (IL-28B $\left.\left(\lambda_{3}\right), \operatorname{IL}-29\left(\lambda_{1}\right)\right)$ and $7 \mathrm{pg} / \mathrm{mL}\left(\mathrm{PGE}_{2}\right)$.

\section{Statistical analysis}

The data depicted in this study are presented as mean \pm SEM. All data sets were verified for normality and, where they were non-parametrically distributed, the dataset was log transformed prior to statistical analysis using GraphPad Prism Version 5 software (California, USA). ELISA and RV titration results were analyzed by 1-way Analysis of Variance (ANOVA) with Bonferroni post-test comparison to their respective control and/or a paired Student's t-test where appropriate. Statistical significance was shown when $\mathrm{p} \leq 0.05$.

\section{Results}

Formoterol induces CAMP and IL-6 from ASMCs

Initial experiments aimed to validate the functionality of our in vitro model by establishing the effects of formoterol on IL-28B, IL-29 (IFN- $\lambda_{1} \&_{3}$ ), cAMP, IL-6, IL-8 and $\mathrm{PGE}_{2}$ production. Levels of IFN- $\lambda$ s were below the detection limit of the ELISA and for this reason were not investigated further (data not shown). As previously reported [29], formoterol induced cAMP and IL-6 (Figure $1 \mathrm{~A}$ and $\mathrm{C}$ ) but not $\mathrm{PGE}_{2}$ compared to vehicle controls (Figure 1B) in ASMCs. The level of IL-8 assessed in this experiment was below the detection limit of the ELISA (data not shown).

\section{Inhibition of $\mathrm{PDE}_{4}$ increased formoterol-induced CAMP, basal CAMP and IL-6 from ASMCs}

The functional activity of piclamilast was confirmed by showing that inhibition of $\mathrm{PDE}_{4}$ further increased formoterol-induced cAMP as well as increasing the basal accumulation of cAMP over 24 hours (Figure 2A \& B). Given that the functional activity of piclamilast was most effective at a concentration of $10^{-6} \mathrm{M}$ this concentration was chosen for subsequent experiments. Piclamilast induced IL-6 release from ASMCs compared to vehicle treatment but did not influence $\mathrm{PGE}_{2}$ release (Figure $2 \mathrm{C}$ \& D). The level of IL- 8 assessed in this experiment was below the detection limit of the ELISA (data not shown). As shown in Figure 3, IL-6 production is additively increased when ASMCs were treated with piclamilast in the presence of formoterol at $10^{-8} \mathrm{M}$ (Figure 3).

\section{$\mathrm{PDE}_{4}$ inhibition modulates TLR agonist-induced cytokine release from ASMCs}

The agonists for TLR 3 and 7/8: poly I:C and imiquimod respectively (surrogates for double stranded and single stranded RNA) were used to treat ASMCs in order to investigate the effects of $\mathrm{PDE}_{4}$ inhibition on the sequelae of TLR activation. To investigate if the TLR agonists directly modulated cAMP we measured cAMP from ASMCs stimulated with TLR agonists for only 30 minutes, to avoid the confounding effects of autocrine action of mediators such as prostaglandins [11]. We found imiquimod induced cAMP compared to vehicle control but poly I:C did not (Figure 4A). Imiquimod did not induce IL-8 (below detection limit of the ELISA) (data not shown) but induced IL-6 from ASMCs compared to vehicle control and this was additively increased in the presence of piclamilast (Figure 4B). In comparison, poly I:C induced both IL6 and IL-8 in ASMCs compared to vehicle control but piclamilast inhibited poly I:C-induced IL-8, and had no effect on IL-6 (Figure 4C \& D). 

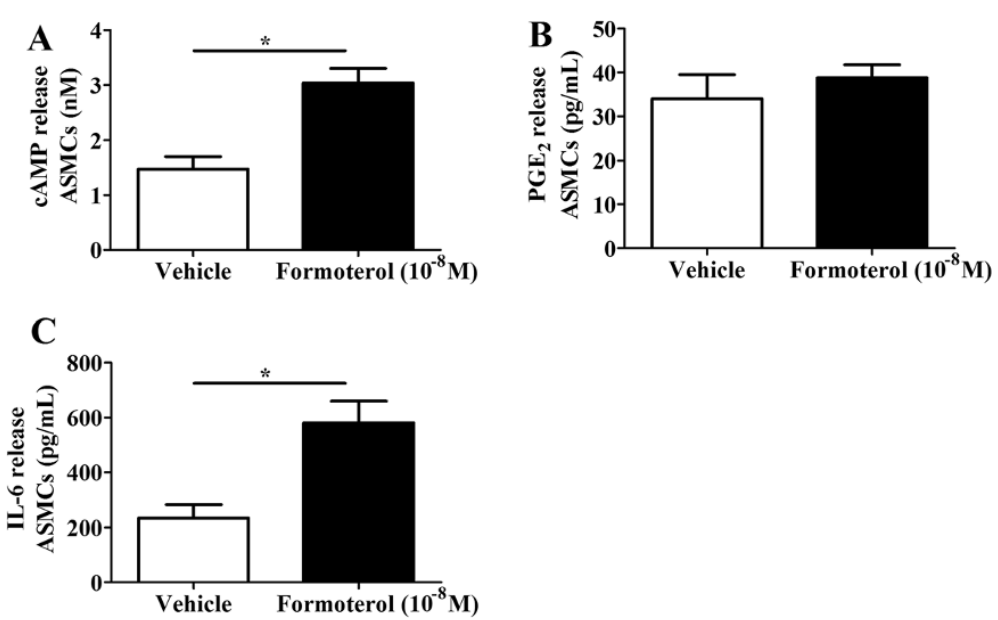

Figure 1 (A-C): Effect of formoterol on CAMP, PGE 2 and IL-6 release from ASMCs. ASMCs were treated with medium (vehicle) or formoterol $\left(10^{-8} M\right)$ and concentration of $\left.\mathbf{A}\right)$ CAMP $(n=5)$ in cell lysates were measured after 5 minutes of stimulation, while $\left.\mathbf{B}\right)$ PGE $(n=5)$ and $\left.\mathbf{C}\right) I L-6$ $(n=7)$ in cell supernatants were measured after 24 hrs. The vehicle control contained $10^{-4} \%(\mathrm{v} / \mathrm{v})$ DMSO. ${ }^{*} \mathrm{p}<0.05$ between groups as indicated by the horizontal lines.

$\mathrm{PDE}_{4}$ inhibitors do not modulate RV-induced cytokines and RV does not induce cAMP

In order to assess the effects of $\mathrm{PDE}_{4}$ inhibition on viral induced inflammation ASMCs were infected with purified live RV-16. RV infection of ASMCs significantly increased the induction of IL-6, IL-8 and $\mathrm{PGE}_{2}$ compared to constitutive release in the presence of vehicle but piclamilast did not alter RV induction of those cytokines (Figure 5A-C). We also found that piclamilast did not alter RV-induced IL-6 (41.2 $\pm 17.3 \mathrm{pg} / \mathrm{mL}$ vs $59.4 \pm$
$23.0 \mathrm{pg} / \mathrm{mL}, \mathrm{n}=4 \mathrm{RV}$ verses $\mathrm{RV}+$ piclamilast) and IL-8 $(437.0 \pm 163.5 \mathrm{pg} / \mathrm{mL}$ vs $531.5 \pm 204.1 \mathrm{pg} / \mathrm{mL}, \mathrm{n}=4 \mathrm{RV}$ verses RV + piclamilast) in HBECs. Furthermore, RV did not induce cAMP within 30 minutes of infection compared to uninfected cells (Figure 5D).

\section{Inhibition of $\mathrm{PDE}_{4}$ does not modulate $\mathrm{RV}$ replication in ASMCs or HBECs}

Subsequently we investigated if inhibition of $\mathrm{PDE}_{4}$ could result in changes to RV replication in ASMCs and in
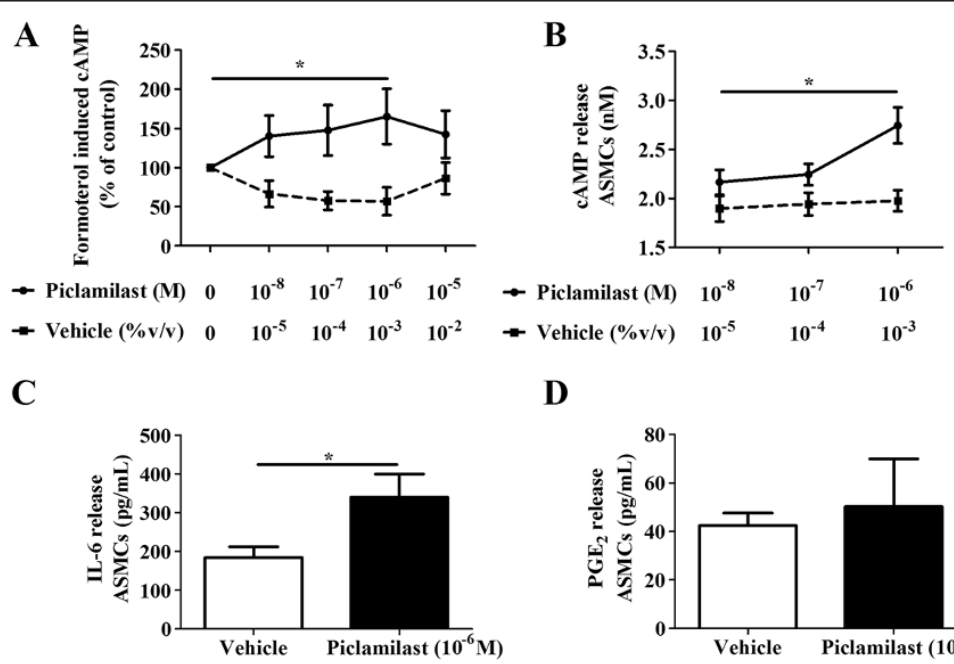

D

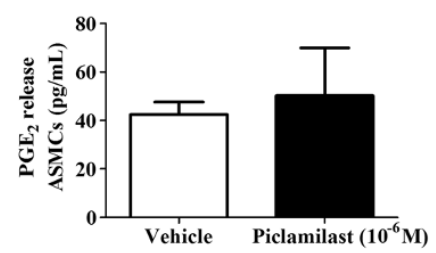

Figure 2 (A-D): Effect of piclamilast on: formoterol-induced CAMP, accumulated CAMP, IL-6 and PGE 2 release from ASMCs.

Concentration of $\mathbf{A}$ ) formoterol-induced CAMP $(n=6)$ in cell lysates were measured after $1 \mathrm{hr}$ pre-treatment with piclamilast $\left(10^{-8}-10^{-5} \mathrm{M}\right)$ or corresponding vehicle $\left(10^{-5}-10^{-2} \%(\mathrm{v} / \mathrm{V})\right.$ DMSO) followed by 5 minutes of stimulation with formoterol at $10^{-8} \mathrm{M}$. Concentration of $\left.\mathbf{B}\right)$ accumulated $\operatorname{CAMP}(n=5)$ in cell lysates were measured after $24 \mathrm{hr}$ pre-treatment with piclamilast $\left(10^{-8}-10^{-6} \mathrm{M}\right)$ or corresponding vehicle $\left(10^{-5}-10^{-3} \%(\mathrm{~V} / \mathrm{V})\right.$ DMSO). Concentrations of $\mathbf{C}) \mathrm{IL}-6(n=17)$ and $\mathbf{D}) \mathrm{PGE}_{2}(\mathrm{n}=4)$ in cell supernatants were collected at 24 hrs from ASMCs treated with vehicle $\left(10^{-3} \%(\mathrm{~V} / \mathrm{V}) \mathrm{DMSO}\right)$ or piclamilast $\left(10^{-6} \mathrm{M}\right) .{ }^{*} \mathrm{p}<0.05$ between groups is compared to the control or the least concentration of piclamilast as indicated by the horizontal lines. 


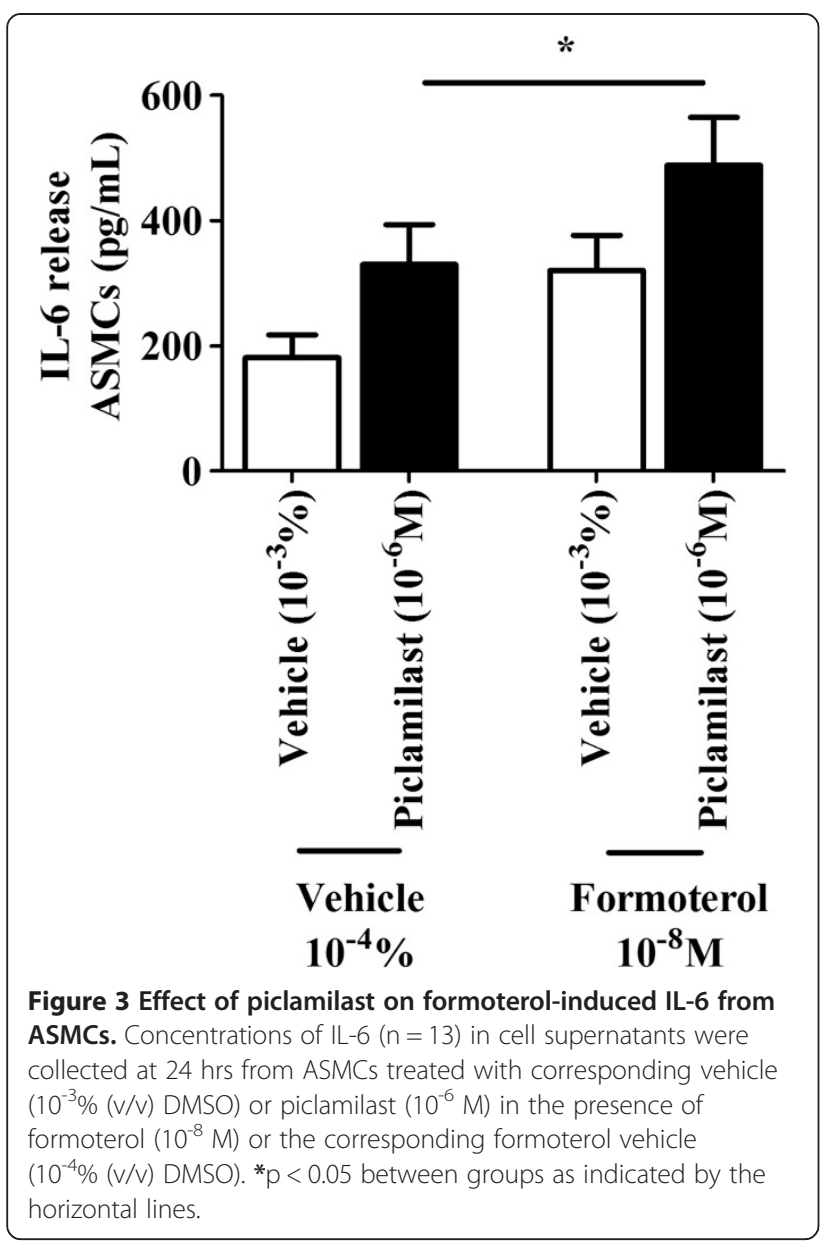

HBECs as HBECs are a source of antiviral IFNs which may interfere with RV replication. Piclamilast did not alter the virion number in either cell type (Figure 6A \& B).

\section{Discussion}

Virus-induced exacerbations of COPD constitute a significant health burden and its management is hampered by the lack of effective therapies. The mechanism by which exacerbations occur remains undefined however it is likely due to virus-induced inflammation. Given that $\mathrm{PDE}_{4}$ inhibitors have been clinically shown to have antiinflammatory effects in patients with COPD they present a potential therapy during these exacerbations. In the present study we investigated whether the $\mathrm{PDE}_{4}$ inhibitor piclamilast could alter virus-induced cytokine release and replication. The novel findings of this study reveal that although $\mathrm{PDE}_{4}$ inhibitors may not influence RVinduced cytokine release and replication in ASMCs, a potential mechanism by which $\mathrm{PDE}_{4}$ inhibitors may be anti-inflammatory could be via the increase of IL- 6 and inhibition of IL-8 induction, resulting in reduced neutrophil numbers under inflammatory conditions.
In the present study we initially established that IL-6 induction is regulated by CAMP by showing that inducing cAMP using formoterol or inhibiting cAMP hydrolysis using piclamilast positively induces IL-6 and cAMP, but not IL-8 or PGE 2 , from ASMCs. Although the findings with IL-6 induction are consistent with others [16] we did not observe any effects on IL-8 release from ASMCs. Pang et al. showed that the $\beta_{2}$-AR agonists salbutamol and salmeterol induced IL-8 release from ASMCs at concentrations greater than $10^{-6} \mathrm{M}$, which were at least 2 logs greater than those used in the present study [20]. At such high concentrations it is possible that transcription factors other than CREB could be activated [30] suggesting that at least in ASMCs, IL-8 transcription may not involve cAMP but rather cross talk between other transcription factors. Furthermore, the lack of change in $\mathrm{PGE}_{2}$ levels may be the result of being dominantly regulated by alternative signalling pathways such as NF- $\kappa$ B [31]. Since PDEs regulate cAMP signalling which in turn can regulate immunomodulatory cytokines, we then investigated whether manipulation of this pathway with the $\mathrm{PDE}_{4}$ inhibitor piclamilast could result in antiviral or anti-inflammatory effects on ASMCs during viral-induced inflammation.

Virus replication generally involves the transcription of viral genomic material to produce new viral progeny. The cells of the innate immune system have evolved to detect foreign viral particles through TLRs. Activation of TLRs result in the increase of COX-2 induced $\mathrm{PGE}_{2}$ which subsequently increase cAMP levels and leads to increased IL-6 and IL-8 [11,32,33]. Therefore, in order to assess whether piclamilast could modulate virusinduced inflammation via TLR activation, analogues of TLRs such as poly I:C and imiquimod were used to stimulate TLRs 3 and 7/8 respectively on ASMCs.

Imiquimod only induced cAMP and IL-6 release from ASMCs and the addition of piclamilast increased levels of IL-6, most likely by extending cAMP activity, similar to those effects of formoterol and piclamilast. Interestingly, poly I:C induced both IL-6 and IL-8 release from ASMCs but did not induce cAMP. This suggests that both cytokines may also be regulated by signalling mechanisms other than cAMP, such as NF- KB and MAPK signalling [34]. On the other hand piclamilast reduced poly I:C-induced IL-8 release which was similar to the findings by Murphy et al. using cilomilast, a PDE4 inhibitor, on HBECs obtained from patients with bronchiolitis obliterans syndrome [24]. Unlike corticosteroids, the sequelae of increasing cAMP is not pan inhibition of cytokine release, for example in ASMCs others have shown that some but not all IL-1 $\beta$-induced cytokines are inhibited as a consequence of stimulating cAMP production [35]. We speculate the mechanism behind inhibition of poly I:C-induced IL- 8 by $\mathrm{PDE}_{4}$ 
A
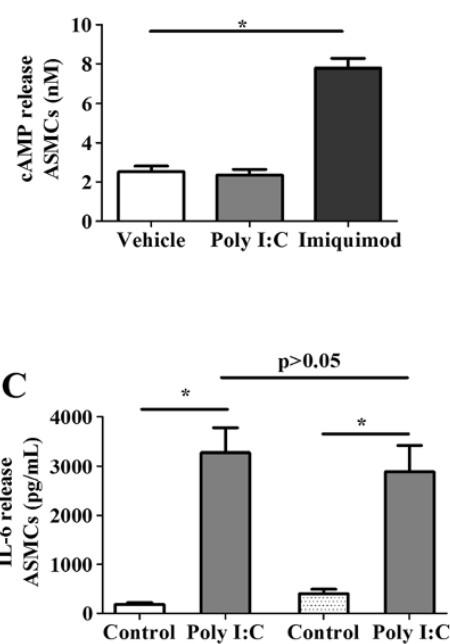

Vehicle
Piclamilast $10^{-6} \mathrm{M}$
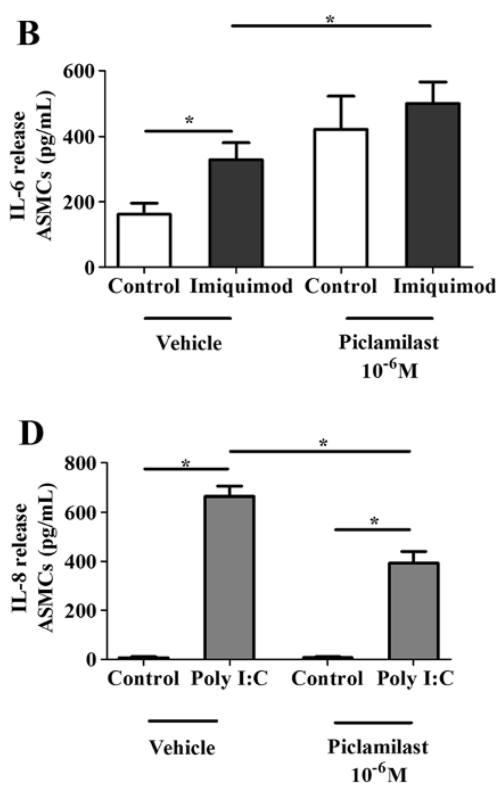

Figure 4 (A-D): Effect of imiquimod and poly I:C on CAMP, IL-6 and IL-8 release from ASMCs in the presence or absence of piclamilast. Concentration of $\mathbf{A}) \operatorname{CAMP}(n=6)$ in cell lysates were measured after 30 minutes of stimulation with imiquimod $(50 \mu \mathrm{g} / \mathrm{mL})$ and poly l:C $(50 \mu \mathrm{g} /$ $\mathrm{mL}$ ). Concentrations of IL-6 (B, C) and IL-8 (D) in cell supernatants were collected at 24 hrs from ASMCs treated with control (vehicle); imiquimod $(5 \mu \mathrm{g} / \mathrm{mL})(\mathrm{n}=10)$ and poly l:C $(5 \mu \mathrm{g} / \mathrm{mL})(\mathrm{n}=9)$ in the presence of vehicle or piclamilast $\left(10^{-6} \mathrm{M}\right)$. The vehicle control consisted of $10^{-3} \%(\mathrm{~V} / \mathrm{v})$ DMSO. ${ }^{*} p<0.05$ between groups as indicated by the horizontal lines.
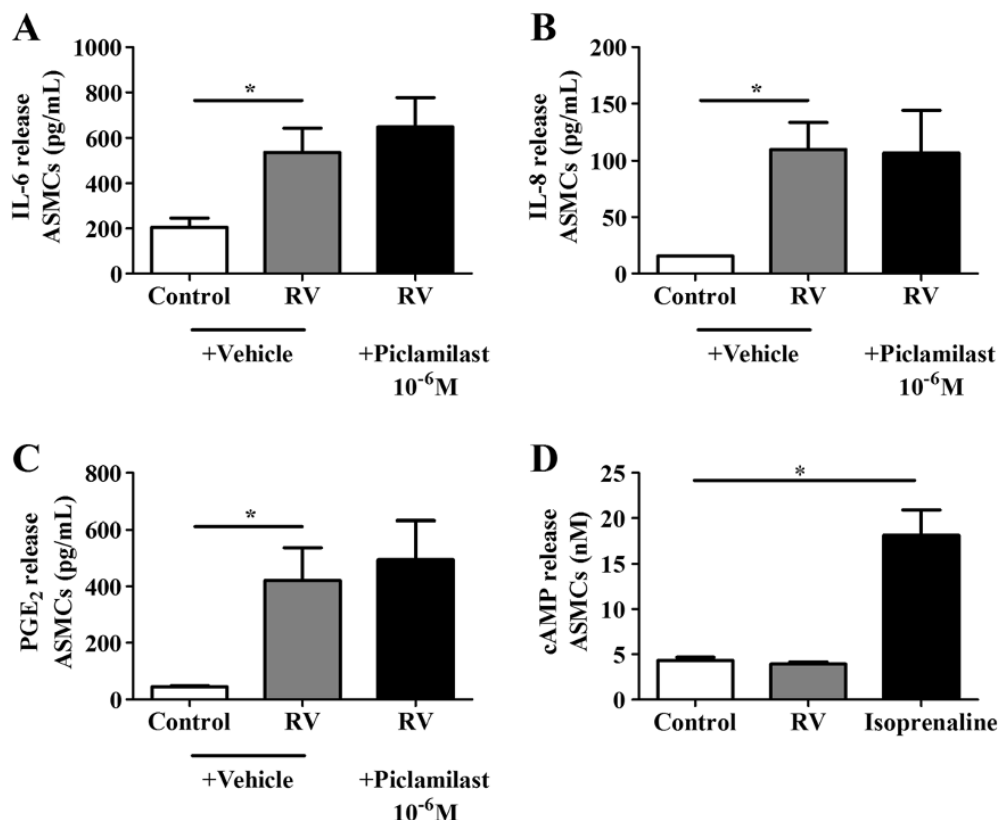

Figure 5 (A-D): Effect of RV-16 on IL-6, IL-8 and PGE 2 release from ASMCs in the presence or absence of piclamilast. Concentrations of A) $I L-6(n=7), B) \| L-8(n=7)$ and C) PGE $(n=4)$ in cell supernatants were collected at 24 hrs from ASMCs treated with control and RV (MOI = 1) in the presence of vehicle or piclamilast $\left(10^{-6} \mathrm{M}\right)$. Concentration of D) CAMP $(n=6)$ in cell lysates were measured after 30 minutes of infection $(\mathrm{MOI}=1)$ or stimulation with the positive control isoprenaline $\left(10^{-7} \mathrm{M}\right)$. The vehicle controls contained $10^{-3} \%(\mathrm{v} / \mathrm{v}) \mathrm{DMSO}$. ${ }^{*} \mathrm{p}<0.05$ between groups as indicated by the horizontal lines. 

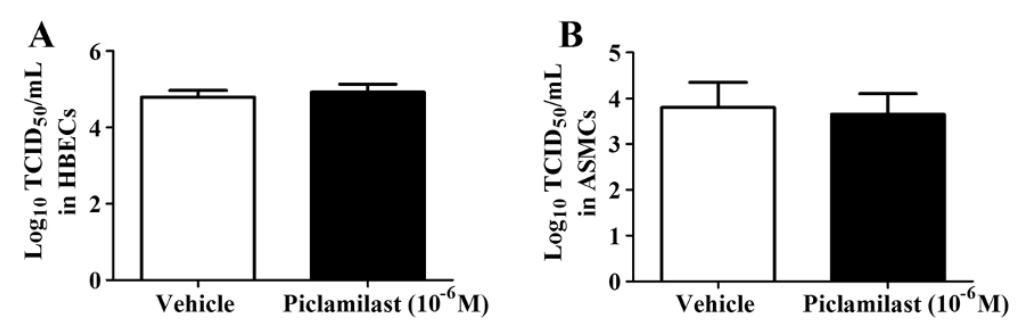

Figure 6 (A-B): Piclamilast did not modulate RV replication in HBECs and ASMCs. A) HBECs $(n=4)$ and B) ASMCs $(n=5)$ were infected with $\mathrm{RV}$ at an $\mathrm{MOI}=1$ in the presence of vehicle $\left(10^{-3} \%(\mathrm{~V} / \mathrm{V}) \mathrm{DMSO}\right)$ or piclamilast $\left(10^{-6} \mathrm{M}\right)$ and $\mathrm{RV}$ concentration in cell supernatants were quantified after 24 hours.

inhibition might be via cAMP sequestration of the NF$\mathrm{\kappa B}$ pathway downstream of immuno-receptors as was shown to be true in splenic B lymphocytes [36]. IL-6 is a cytokine with multiple biological roles and traditionally considered a pro-inflammatory marker as its levels are often detected to be increased in inflammatory diseases such as asthma [37]. However the biological functions of IL-6 do not make it entirely a contributor to inflammation as more recently other roles which contribute to the resolution of the innate immune response, such as reducing neutrophil trafficking during acute inflammation, have been uncovered [38]. Since reports have shown that IL- 6 can limit the recruitment of neutrophils and oppositely, IL-8 can induce neutrophil chemotaxis $[38,39]$, our results suggest that the increase in IL-6 and reduction of IL-8 cytokine release by $\mathrm{PDE}_{4}$ inhibition during inflammatory circumstances may be important in the anti-inflammatory mechanism of action of $\mathrm{PDE}_{4}$ inhibitors.

In order to assess the effects of $\mathrm{PDE}_{4}$ inhibition on virus infection, as opposed to the use of viral surrogates, ASMCs were infected with RV-16. RV infection of ASMCs resulted in the induction of inflammatory cytokines and lipids including IL6, IL- 8 and PGE $_{2}$ but were unaffected by $\mathrm{PDE}_{4}$ inhibition. Similarly, RV infection of HBECs which have been shown by others to induce IL-6 and IL-8 [40] were unaffected by $\mathrm{PDE}_{4}$ inhibition. Significantly our study also shows that RV infection in ASMCs did not induce cAMP in an acute setting. In a longer incubation, RV may be able to induce cAMP through its replication, as increased viral replication will result in the activation of TLRs and subsequently the induction of cAMP and cytokines by COX-2 induced prostaglandins [11]. Since RV infection causes sensitization of adenylyl cyclase [18] these prostaglandins can further induce cAMP induction in an autocrine manner as we have previously shown [11]. Although we initially established that cAMP induction correlates to increased levels of IL- 6 in our study there are limitations that should be noted. Because cAMP signalling occurs earlier than protein production, it would be interesting to quantify the amount of cAMP that is required to induce a certain amount of IL- 6 at 24 hours; however this will be difficult in the context of RV infection. The reason for this being measurements of cAMP over 24 hours will be confounded by factors such as RV-induced cell death and the influence of other induced mediators such as prostaglandins. Nevertheless, these results suggest that $\mathrm{RV}$-induced inflammation is complex, with the activation of multiple inflammatory signal pathways such as NF- $\kappa \mathrm{B}, \mathrm{cAMP}$ signalling and prostaglandins pathways, as TLRs are activated during increased RV replication $[10,19,41]$.

RV infections are major contributors to exacerbations of COPD [42,43]. Methods to prevent or treat these infections could aid in reducing COPD exacerbations, however there are currently no anti-rhinoviral pharmacotherapies on the market. Anti-inflammatory corticosteroids are already widely used in the treatment of airway inflammation. They have demonstrated inhibitory effects on RV replication in epithelial cell culture possibly by reducing the expression of the RV entry receptor ICAM-1 $[44,45]$. However this effect appears to be cell dependent as corticosteroids do not alter RV replication in cultured airway fibroblasts [41]. Clinically, corticosteroids do not appear to have an effect on the symptoms of the common cold and lead to prolonged RV replication in the airways [46]. Therefore, improved medications are needed for both anti-inflammatory and anti-viral treatments in exacerbations of COPD and asthma.

$\mathrm{PDE}_{4}$ inhibitors inhibit TNF- $\alpha$ stimulated expression of ICAM-1 in fibroblasts suggesting that, as with corticosteroids, they may be able to inhibit RV infection by reducing ICAM-1 expression [47]. The present study showed that the $\mathrm{PDE}_{4}$ inhibitor piclamilast had no effect on RV replication, demonstrating that either ASMCs do not have the ability to inhibit RV replication or that cAMP signalling does not regulate the innate anti-viral response to RV. IFNs are a major anti-viral group of cytokines and, in support of the former possibility, levels of IFN- $\lambda$ measured in cell supernatants of infected ASMCs in this study were below the detection limit of the ELISA. Although Calven et al. showed that infection 
with RV-1B induced significant mRNA levels of the type I IFNs (IFN- $\beta$ ) and IFN- $\lambda_{1}$ in bronchial ASM, their corresponding protein levels post-infection in cell supernatants were reported only just at detection level. Similarly, others have shown that primary human ASMCs and lung fibroblasts do not produce IFNs in response to RV infection $[10,41,48]$. In this regard, the biological significance of the level of induced IFN proteins from ASMCs and, whether ASMCs have the ability to produce IFN proteins, remains controversial. If ASMCs do not produce IFNs then it is highly likely that they do not possess the ability to inhibit RV replication in the absence of other cell types. Bronchial epithelial cells on the other hand express and produce IFNs in response to RV infection, and in a multicellular environment may have the ability to induce antiviral effects on other cells such as ASMCs and fibroblasts [49,50]. However, in this study piclamilast also had no effect on RV replication in HBECs, highlighting the fact that cAMP signalling may not be regulating the innate anti-viral response to $\mathrm{RV}$, or a more complex system underlies regulation of viral replication than just IFNs.

There is a vast amount of evidence to suggest that cells from different disease states respond and behave differently to each other. For example asthmatic ASMCs and epithelial cells produce more IL-6 and less IFNs respectively in response to RV infection compared to non asthmatic cells $[10,49]$. In this study we examined the effects of $\mathrm{PDE}_{4}$ inhibitors on viral replication and inflammation in a pooled group of primary human ASMCs obtained from various patients with respiratory diseases. Since there were insufficient cells for each disease group we were not able to compare cell responses between diseased populations. Therefore future studies looking at the differences between healthy and COPD or asthmatic cells may further reveal whether the role of $\mathrm{PDE}_{4}$ during $\mathrm{RV}$, as well as other respiratory viral infections, is altered in different diseases.

Additionally, in our study we showed that ASMCs produce levels of IL-6 in response to formoterol which is further increased by $\mathrm{PDE}_{4}$ inhibition. Although this is consistent with the findings by other in vitro studies [29] intuitively this would suggest that $\beta_{2}$-AR agonists create a pro-inflammatory environment which could be detrimental to the inflammatory conditions already present in diseases such as COPD. However this is not the case, because in in vivo mouse model studies of inflammatory lung disorders, $\beta_{2}$-AR agonists have been shown to have anti-inflammatory effects and reduce IL-6 levels [51,52]. While clinically, $\beta_{2}$-AR agonists have been shown to be very effective therapies in inflammatory respiratory diseases such as asthma when combined with corticosteroids [53]. Why in vivo and in vitro responses differ is not known however it is likely due to the combined multicellular interaction and response to $\beta_{2}$-AR agonists, thus highlighting a potential limitation of our monocellular study. In tissue where all cells are present, it is likely that the pool of mediators will complement each other in a physiological interaction. For this reason it is unlikely that physiologically any single group of cells would be more or less important than another in terms of cytokine induction. Therefore future studies could expand on this study using in vitro co-culture systems and/or in vivo mouse models to better demonstrate a more multicellular interactive response to $\beta_{2}$-AR agonists and $\mathrm{PDE}_{4}$ inhibitors.

\section{Conclusion}

A characteristic feature of COPD is inflammation of the lungs with increased levels of IL-8 detected in patient bronchoalveolar fluids compared to non COPD patients [54]. In COPD patients $\mathrm{PDE}_{4}$ inhibitors have been shown to be anti-inflammatory by reducing neutrophils and eosinophils in their sputum [23]. Others have also shown that $\mathrm{PDE}_{4}$ inhibitors can reduce basal inflammatory levels of IL-8 in HBECs obtained from patients with the severe inflammatory respiratory disorder: bronchiolitis obliterans syndrome [24]. Our study suggests that this reduction of neutrophils may be due to the ability of $\mathrm{PDE}_{4}$ inhibitors to modulate IL-6 and also IL-8 levels in ASMCs and therefore limit neutrophil recruitment [38]. $\mathrm{PDE}_{4}$ inhibitors have been shown to synergistically increase the effects of $\beta_{2}$-AR agonists, and dual specific $\mathrm{PDE}_{4 / 3}$ inhibitors have been shown to cause acute short acting bronchodilation in people with asthma [55-57]. We demonstrated for the first time that $\mathrm{PDE}_{4}$ inhibitors do not alter RV replication or the inflammatory response to RV infections in ASMCs and HBECs. Because $\mathrm{PDE}_{4}$ inhibitors do not modulate RV-induced inflammation or replication this study suggests $\mathrm{PDE}_{4}$ inhibitors may be safe to supplement bronchodilating medication during RV-induced exacerbations of COPD and asthma as well as having other beneficial effects of reducing inflammatory neutrophils during exacerbations of COPD caused by bacteria or viruses other than RV.

\section{Competing interests \\ The authors declare that they have no competing interests.}

\section{Authors' contributions}

DV and BGGO designed these studies with contribution from $L M, J L B$ and JKB; DV, MD and PJ carried out the experiments, analyzed the data and interpreted the results of the experiments; DV prepared and drafted the manuscript; DV, JLB, JKB, LM and BGGO edited, revised and approved the final version of the manuscript.

\section{Acknowledgements}

We acknowledge the collaborative effort of the cardiopulmonary transplant team and the pathologists at St Vincent's Hospital, Sydney and the thoracic physicians and pathologists at Royal Prince Alfred Hospital, Concord Repatriation General Hospital and Strathfield Private Hospital and Rhodes Pathology, Sydney. JKB is supported by a National Health and Medical 
Research Council Career Development Fellowship \#1032695. JLB is supported by a National Health and Medical Research Council Senior Principal Research Fellowship \#571098. BGGO is supported by a National Health and Medica Research Council Career Development Fellowship APP1026880.

\section{Author details}

${ }^{1}$ Woolcock Institute of Medical Research, Sydney, Australia. ${ }^{2}$ Respiratory Research Group, Discipline of Pharmacology, The University of Sydney, Sydney, New South Wales, Australia. ${ }^{3}$ Department of Thoracic Medicine, Concord Repatriation General Hospital, Concord, New South Wales, Australia.

Received: 8 August 2013 Accepted: 11 November 2013

Published: 15 November 2013

\section{References}

1. Kodimuthali A, Jabaris SSL, Pal M: Recent advances on phosphodiesterase 4 inhibitors for the treatment of asthma and chronic obstructive pulmonary disease. J Med Chem 2008, 51:5471-5489.

2. Celli BB PJ: Exacerbations of chronic obstructive pulmonary disease. Eur Respir J 2007, 29:1224-1238.

3. Seemungal TA, Donaldson GC, Bhowmik A, Jeffries DJ, Wedzicha JA: Time course and recovery of exacerbations in patients with chronic obstructive pulmonary disease. Am J Respir Crit Care Med 2000 161:1608-1613.

4. Potena A, Caramori G, Casolari P, Contoli M, Johnston SL, Papi A: Pathophysiology of viral-induced exacerbations of COPD. Int I Chron Obstruct Pulmon Dis 2007, 2:477-483.

5. Warren HS: Toll-like receptors. Crit Care Med 2005, 33:S457.

6. Sabroe I, Whyte M: Toll-like receptor (TLR)-based networks regulate neutrophilic inflammation in respiratory disease. Biochem Soc Trans 2007, 35:1492-1495.

7. Hewson CA, Jardine A, Edwards MR, Laza-Stanca V, Johnston SL: Toll-like receptor 3 is induced by and mediates antiviral activity against rhinovirus infection of human bronchial epithelial cells. J Virol 2005, 79:12273.

8. Slater L, Bartlett NW, Haas JJ, Zhu J, Message SD, Walton RP, Sykes A, Dahdaleh S, Clarke DL, Belvisi MG, et al: Co-ordinated role of TLR3, RIG-I and MDA5 in the innate response to rhinovirus in bronchial epithelium. PLoS Pathog 2010, 6:e1001178.

9. Papadopoulos NG, Bates PJ, Bardin PG, Papi A, Leir SH, Fraenkel DJ, Meyer J, Lackie PM, Sanderson G, Holgate ST, Johnston SL: Rhinoviruses infect the lower airways. J Infect Dis 2000, 181:1875-1884.

10. Oliver B, Johnston S, Baraket M, Burgess J, King N, Roth M, Lim S, Black J: Increased proinflammatory responses from asthmatic human airway smooth muscle cells in response to rhinovirus infection. Respir Res 2006, 7:71.

11. Van Ly D, Faiz A, Jenkins C, Crossett B, Black JL, McParland B, Burgess JK, Oliver BG: Characterising the mechanism of airway smooth muscle beta2 adrenoceptor desensitization by rhinovirus infected bronchial epithelial cells. PLoS One 2013, 8:e56058.

12. Black JL, Panettieri RA Jr, Banerjee A, Berger P: Airway smooth muscle in asthma: just a target for bronchodilation? Clin Chest Med 2012, 33:543-558.

13. Panettieri RA Jr: Airway smooth muscle: immunomodulatory cells? Allergy Asthma Proc 2004, 25:381-386

14. Daniel PB, Walker WH, Habener JF: Cyclic AMP signaling and gene regulation. Annu Rev Nutr 1998, 18:353-383.

15. Strandberg K, Palmberg L, Larsson K: Effect of formoterol and salmeterol on IL-6 and IL-8 release in airway epithelial cells. Respir Med 2007, 101:1132-1139.

16. Ammit AJ, Lazaar AL, Irani C, O'Neill GM, Gordon ND, Amrani Y, Penn RB, Panettieri RA Jr: Tumor necrosis factor-alpha-induced secretion of RANTES and interleukin-6 from human airway smooth muscle cells: modulation by glucocorticoids and beta-agonists. Am J Respir Cell Mol Biol 2002, 26:465-474.

17. Wu YL, Wiltbank MC: Transcriptional regulation of the cyclooxygenase-2 gene changes from protein kinase (PK) a- to PKC-dependence after luteinization of granulosa cells. Biol Reprod 2002, 66:1505-1514.

18. Billington CK, Pascual RM, Hawkins ML, Penn RB, Hall IP: Interleukin-1 beta and rhinovirus sensitize adenylyl cyclase in human airway smoothmuscle cells. Am J Respir Cell Mol Biol 2001, 24:633-639.
19. Edwards MR, Haas J, Panettieri RA Jr, Johnson M, Johnston SL: Corticosteroids and beta2 agonists differentially regulate rhinovirusinduced interleukin-6 via distinct Cis-acting elements. J Biol Chem 2007, 282:15366-15375.

20. Pang L, Knox AJ: Synergistic inhibition by beta(2)-agonists and corticosteroids on tumor necrosis factor-alpha-induced interleukin-8 release from cultured human airway smooth-muscle cells. Am J Respir Cell Mol Biol 2000, 23:79-85.

21. Roebuck KA: Regulation of interleukin-8 gene expression. J Interferon Cytokine Res 1999, 19:429-438.

22. Trian T, Burgess JK, Niimi K, Moir LM, Ge Q, Berger P, Liggett SB, Black JL, Oliver BG: Beta2-agonist induced CAMP is decreased in asthmatic airway smooth muscle due to increased PDE4D. PLoS One 2011, 6:e20000.

23. Grootendorst DC, Gauw SA, Verhoosel RM, Sterk PJ, Hospers JJ, Bredenbröker D, Bethke TD, Hiemstra PS, Rabe KF: Reduction in sputum neutrophil and eosinophil numbers by the PDE4 inhibitor roflumilast in patients with COPD. Thorax 2007, 62:1081.

24. Murphy DM, Ward C, Forrest IA, Pritchard G, Jones D, Stovold R, Fisher AJ, Cawston TE, Lordan JL, Corris PA: The phosphodiesterase type IV inhibitor cilomilast decreases pro-inflammatory cytokine production from primary bronchial epithelial cells in lung transplantation patients. J Heart Lung Transplant 2006, 25:1436-1440.

25. Calverley PM, Anderson JA, Celli B, Ferguson GT, Jenkins C, Jones PW, Yates $J C$, Vestbo J: Salmeterol and fluticasone propionate and survival in chronic obstructive pulmonary disease. N Engl J Med 2007, 356:775-789.

26. Hatzelmann A, Morcillo EJ, Lungarella G, Adnot S, Sanjar S, Beume R, Schudt $\mathrm{C}$, Tenor $\mathrm{H}$ : The preclinical pharmacology of roflumilast a selective, oral phosphodiesterase 4 inhibitor in development for chronic obstructive pulmonary disease. Pulm Pharmacol Ther 2010, 23:235-256.

27. Johnson PR, Roth M, Tamm M, Hughes M, Ge Q, King G, Burgess JK, Black $J$ : Airway smooth muscle cell proliferation is increased in asthma. Am J Respir Crit Care Med 2001, 164:474-477.

28. Bartlett NW, Walton RP, Edwards MR, Aniscenko J, Caramori G, Zhu J, Glanville N, Choy KJ, Jourdan P, Burnet J, et al: Mouse models of rhinovirus-induced disease and exacerbation of allergic airway inflammation. Nat Med 2008, 14:199-204.

29. Niimi K, Ge Q, Moir LM, Ammit AJ, Trian T, Burgess JK, Black JL, Oliver BG: Beta2-agonists upregulate PDE4 mRNA but not protein or activity in human airway smooth muscle cells from asthmatic and nonasthmatic volunteers. Am J Physiol Lung Cell Mol Physiol 2012, 302:L334-L342.

30. Manetsch M, Rahman MM, Patel BS, Ramsay EE, Rumzhum NN, Alkhouri H, Ge Q, Ammit AJ: Long-acting beta2-agonists increase fluticasone propionate-induced mitogen-activated protein kinase phosphatase 1 (MKP-1) in airway smooth muscle cells. PLoS One 2013, 8:e59635.

31. Nakao S, Ogtata Y, Shimizu E, Yamazaki M, Furuyama S, Sugiya H: Tumor necrosis factor alpha (TNF-alpha)-induced prostaglandin E2 release is mediated by the activation of cyclooxygenase-2 (COX-2) transcription via NFkappaB in human gingival fibroblasts. Mol Cell Biochem 2002, 238:11-18.

32. Penn RB, Panettieri RA Jr, Benovic JL: Mechanisms of acute desensitization of the beta2AR-adenylyl cyclase pathway in human airway smooth muscle. Am J Respir Cell Mol Biol 1998, 19:338-348.

33. Van Ly D, Burgess JK, Brock TG, Lee TH, Black JL, Oliver BG: Prostaglandins but not leukotrienes alter extracellular matrix protein deposition and cytokine release in primary human airway smooth muscle cells and fibroblasts. Am J Physiol Lung Cell Mol Physiol 2012, 303:L239-L250.

34. Jiang Z, Zamanian-Daryoush M, Nie H, Silva AM, Williams BR, Li X: Poly(I-C)induced Toll-like receptor 3 (TLR3)-mediated activation of NFkappa B and MAP kinase is through an interleukin-1 receptor-associated kinase (IRAK)-independent pathway employing the signaling components TLR3-TRAF6-TAK1-TAB2-PKR. J Biol Chem 2003, 278:16713-16719.

35. Hallsworth MP, Twort CH, Lee TH, Hirst SJ: Beta(2)-adrenoceptor agonists inhibit release of eosinophil-activating cytokines from human airway smooth muscle cells. Br J Pharmacol 2001, 132:729-741.

36. Minguet $S$, Huber M, Rosenkranz L, Schamel WW, Reth M, Brummer T: Adenosine and CAMP are potent inhibitors of the NF-kappa B pathway downstream of immunoreceptors. Eur J Immunol 2005, 35:31-41.

37. Neveu WA, Allard JL, Raymond DM, Bourassa LM, Burns SM, Bunn JY, Irvin CG, Kaminsky DA, Rincon M: Elevation of IL- 6 in the allergic asthmatic airway is independent of inflammation but associates with loss of central airway function. Respir Res 2010, 11:28. 
38. Fielding CA, McLoughlin RM, McLeod L, Colmont CS, Najdovska M, Grail D, Ernst M, Jones SA, Topley N, Jenkins BJ: IL-6 regulates neutrophil trafficking during acute inflammation via STAT3. J Immuno/ 2008, 181:2189-2195

39. Hammond ME, Lapointe GR, Feucht PH, Hilt S, Gallegos CA, Gordon CA, Giedlin MA, Mullenbach G, Tekamp-Olson P: IL-8 induces neutrophil chemotaxis predominantly via type I IL-8 receptors. J Immunol 1995, 155:1428-1433.

40. Subauste MC, Jacoby DB, Richards SM, Proud D: Infection of a human respiratory epithelial cell line with rhinovirus. Induction of cytokine release and modulation of susceptibility to infection by cytokine exposure. J Clin Invest 1995, 96:549-557.

41. Van Ly D, King NJ, Moir LM, Burgess JK, Black JL, Oliver BG: Effects of beta (2) agonists, corticosteroids, and novel therapies on rhinovirus-induced cytokine release and rhinovirus replication in primary airway fibroblasts. J Allergy (Cairo) 2011, 2011:457169.

42. Makela MJ, Puhakka T, Ruuskanen O, Leinonen M, Saikku P, Kimpimaki M, Blomqvist S, Hyypia T, Arstila P: Viruses and bacteria in the etiology of the common cold. J Clin Microbiol 1998, 36:539.

43. Papi A, Bellettato CM, Braccioni F, Romagnoli M, Casolari P, Caramori G, Fabbri LM, Johnston SL: Infections and airway inflammation in chronic obstructive pulmonary disease severe exacerbations. Am J Respir Crit Care Med 2006, 173:1114-1121.

44. Ghildyal R, Dagher H, Donninger H, De Silva D, Li X, Freezer NJ, Wilson JW, Bardin PG: Rhinovirus infects primary human airway fibroblasts and induces a neutrophil chemokine and a permeability factor. J Med Virol 2005, 75:608-615.

45. Suzuki T, Yamaya M, Sekizawa K, Yamada N, Nakayama K, Ishizuka S, Kamanaka M, Morimoto T, Numazaki Y, Sasaki H: Effects of dexamethasone on rhinovirus infection in cultured human tracheal epithelial cells. Am J Physiol Lung Cellular Mol Physiol 2000, 278:L560

46. Puhakka T, Makela MJ, Malmstrom K, Uhari M, Savolainen J, Terho EO, Pulkkinen M, Ruuskanen O: The common cold: effects of intranasal fluticasone propionate treatment. J Allergy Clin Immunol 1998, 101:726-731.

47. Sabatini F, Petecchia L, Boero S, Silvestri M, Klar J, Tenor H, Beume R, Hatzelmann A, Rossi G: A phosphodiesterase 4 inhibitor, roflumilast $\mathrm{N}$-oxide, inhibits human lung fibroblast functions in vitro. Pulm Pharmacol Ther 2010, 23:283-291.

48. Bedke N, Haitchi HM, Xatzipsalti M, Holgate ST, Davies DE: Contribution of bronchial fibroblasts to the antiviral response in asthma. J Immuno/ 2009, 182:3660

49. Contoli M: Role of deficient type III interferon- production in asthma exacerbations. Nat Med 2006, 12:1023-1026

50. Wark PAB, Johnston SL, Bucchieri F, Powell R, Puddicombe S, Laza-Stanca V, Holgate ST, Davies DE: Asthmatic bronchial epithelial cells have a deficient innate immune response to infection with rhinovirus. J Exp Med 2005, 201:937

51. Dhingra VK, Uusaro A, Holmes CL, Walley KR: Attenuation of lung inflammation by adrenergic agonists in murine acute lung injury. Anesthesiology 2001, 95:947-953.

52. Bosmann M, Grailer JJ, Zhu K, Matthay MA, Sarma JV, Zetoune FS, Ward PA: Anti-inflammatory effects of beta2 adrenergic receptor agonists in experimental acute lung injury. FASEB J 2012, 26:2137-2144.

53. Ducharme FM, Ni Chroinin M, Greenstone I, Lasserson TJ: Addition of long-acting beta2-agonists to inhaled corticosteroids versus same dose inhaled corticosteroids for chronic asthma in adults and children. Cochrane Database Syst Rev 2010:CD005535.

54. Hollander C, Sitkauskiene B, Sakalauskas R, Westin U, Janciauskiene SM: Serum and bronchial lavage fluid concentrations of IL-8, SLPI, SCD14 and sICAM-1 in patients with COPD and asthma. Respir Med 2007, 101:1947-1953.

55. Au B: Effect of PDE4 inhibitors on zymosan-induced IL-8 release from human neutrophils: synergism with prostanoids and salbutamol. Br J Pharmacol 1998, 123:1260-1266.
56. Brunnee $T$, Engelstatter $R$, Steinijans $V$, Kunkel G: Bronchodilatory effect of inhaled zardaverine, a phosphodiesterase III and IV inhibitor, in patients with asthma. Eur Respir J 1992, 5:982

57. Thomas T, Moir LM, Burgess JK, Liggett SB, Ge Q, Black JL, Oliver BG: Beta2Agonist induced camp is decreased in asthmatic airway smooth muscle due to increased PDE4D. Am J Respir Crit Care Med 2011, 183:A2075.

doi:10.1186/1465-9921-14-127

Cite this article as: Van Ly et al.: Inhibition of phosphodiesterase 4 modulates cytokine induction from toll like receptor activated, but not rhinovirus infected, primary human airway smooth muscle. Respiratory Research 2013 14:127.

\section{Submit your next manuscript to BioMed Central and take full advantage of:}

- Convenient online submission

- Thorough peer review

- No space constraints or color figure charges

- Immediate publication on acceptance

- Inclusion in PubMed, CAS, Scopus and Google Scholar

- Research which is freely available for redistribution 